DOI $10.18307 / 2015.0501$

(C) 2015 by Journal of Lake Sciences

\title{
利用流式细胞仪计数微型浮游生物的方法
}

\author{
李胜男 ${ }^{1,2}$, 王秀娟 ${ }^{1,2}$, 周 建 ${ }^{1,2}$, 孔繁翔 ${ }^{1}$, 史小丽 ${ }^{1 * *}$ \\ ( 1 : 中国科学院南京地理与湖泊研究所湖泊与环境国家重点实验室,南京 210008) \\ (2: 中国科学院大学, 北京 100049)
}

摘 要: 微型浮游生物 (细胞粒径 $<20 \mu \mathrm{m}$ ) 在水生生态系统的物质循环和能量流动中起着重要的作用, 对其丰度的准确 测定是进一步研究微型浮游生物在不同水生生态系统中作用的重要基础. 相对于传统的显微镜检测技术, 流式细胞术不 仅具有分析速度快、灵敏度和准确度高等优点, 而且可以同时测量单个细胞的多个生理参数. 不同类型微型浮游生物流 式细胞术的应用原理是不同的. 对于自养型浮游藻类,主要根据藻体内色素的自发苂光对其进行分辨和计数; 而对于异 养型细菌、原生动物及浮游病毒等,还需借助外源荧光染料对细胞核酸染色后再进行分析. 目前流式细胞术已成为浮游 藻类和异养细菌丰度检测的常规方法, 但是, 由于原生动物具有更大的细胞体积且在自然水体中丰度较低; 而浮游病毒 粒径又太小, 甚至低于光源激发波长, 因此流式细胞术应用一直受到限制, 直到近 10 年来才有相关报道. 本文对运用流式 细胞术计数浮游藻类、浮游细菌、原生动物和浮游病毒的具体原理、方法和进展进行综述, 并对流式细胞仪在未来水生微 生物学领域的应用进行展望.

关键词: 流式细胞仪;浮游植物; 异养细菌;原生动物;病毒;色素;菼光染料

\section{Application of flow cytometry to enumerate small plankton}

\author{
LI Shengnan $^{1,2}$, WANG Xiujuan ${ }^{1,2}$, ZHOU Jian ${ }^{1,2}$, KONG Fanxiang ${ }^{1}$ \& SHI Xiaoli ${ }^{1}$ \\ (1: State Key Laboratory of Lake Science and Environment, Nanjing Institute of Geography and Limnology, Chinese Academy \\ of Sciences, Nanjing 210008, P. R. China) \\ (2: University of Chinese Academy of Sciences, Beijing 100049, P. R. China)
}

\begin{abstract}
The small plankton (cell size less than $20 \mu \mathrm{m}$ ) plays an important role in the circulation of materials and energy flow of the aquatic system. Accurate enumeration of these organisms is the basis for understanding their ecological role in various water bodies. In comparison with microscopy observation, flow cytometry analysis is much more advantageous in terms of speed, sensitivity and accuracy. Moreover, multiple parameters of a single cell could be measured simultaneously using flow cytometry. The protocol to enumerate plankton depends on the type of plankton. Discrimination of several major groups of phytoplankton is mainly based on the differences in the fluorescence properties of their photosynthetic pigments. While for the heterotrophic bacteria, protozoan and viruses, a combination of exogenous fluorochromes staining on cell components (mainly nucleic acids) is required to better characterize different cell groups. Now flow cytometry has become a routine methodology for detecting density of the autotrophic phytoplankton and heterotrophic bacterioplankton. However, it has been only used in quantification of protozoan and viruses in the recent 10 years, for those applications which are much more difficult and complicated for the larger cell size and less abundant densities of protozoan and much smaller cell size (even smaller than the wavelength of the laser light used) of viruses compared to bacterioplankton and small phytoplankton. The different principles and protocols used to discriminate autotrophic phytoplankton, heterotrophic bacteria, protozoan and viruses through flow cytometry were reviewed in detail, and future applications of flow cytometry in aquatic microbiology were also prospected.
\end{abstract}

Keywords: Flow cytometry; phytoplankton; heterotrophic bacteria; protozoan; viruses; pigments; fluorochromes

* 国家自然科学基金项目 (31270507,31070420) 资助. 2014-12-28 收稿; 2015-02-04 收修改稿. 李胜男 (1989 ), 女,博士研究生;E-mail: shengnan1989812@126. com.

** 通信作者;E-mail:xlshi@ niglas. ac.cn. 
微型浮游生物 ( 细胞粒径 $<20 \mu \mathrm{m}$ ) 主要包括浮游藻类、异养细菌、原生动物和浮游病毒等, 是微食物环 的主要组成部分, 在水生生态系统中起着重要作用 ${ }^{[1]}$, 因而对这些微型浮游生物的准确计数就显得尤为重 要. 定量检测其种群动态变化, 不仅有利于研究其种间营养关系, 更有助于探索这些微生物在生物化学地球 循环中所起的重要作用. 传统显微计数方法的优势在于可通过形态特征观察, 对不同类型的微生物分别计 数, 但是, 这种计数方法不仅费时费力, 而且需要丰富的种类鉴定经验, 人为误差较大; 此外, 在实际研究中 由于微生物数量变化迅速, 通常采样频率高, 样品量巨大, 仅通过显微镜计数难以满足研究需求. 而且在很 多研究中常常不需要对这些微生物进行具体的分类计数, 只需测定其类群总量. 流式细胞仪 (flow cytometry) 的应用,使得这些问题迎刃而解.

流式细胞技术是指对处在快速直线流动状态中的单细胞或生物颗粒进行多参数的、快速的定量分析和 分选的技术. 样品中的悬浮颗粒 (如微型浮游生物细胞) 在鞘液的包被下呈单行排列, 依次流经检测区域. 在 检测区, 激光束照射在通过的颗粒上, 一方面由于颗粒大小和内部结构等的差异向四周发射不同角度的散 射光; 另一方面颗粒吸收光能激发不同波长或颜色的苂光, 而这些散射光和苂光都能被不同的检测器检测 产生信号. 散射光主要分为前向角散射光 (forward scatter light, FSC) 和侧向角散射光 ( side scatter light, SSC), FSC 与被测颗粒的直径密切相关, 基本上可表征颗粒的大小; 而 SSC 受颗粒的内部结构如细胞质折射 率、细胞器等影响重大, 反映的是细胞的复杂程度. 发射苂光经一系列双色反射镜和带通滤光片的分离, 形 成多个不同波长的苂光信号, 这些苂光信号的强弱代表了细胞颗粒内含有相关荧光物质的浓度, 如浮游植 物细胞中所含能自发荧光色素的浓度. 通过这些表征颗粒不同性质的光信号就可将不同类型的颗粒区分开 来. 相对于显微计数, 流式细胞术可以同时对多种不同大小、不同苂光特性的细胞进行计数, 不仅准确性高, 而且能测得更多的参数. 此外, 流式细胞术还具有分析速度快、精确度高、样品预处理更简单等优点.

流式细胞仪主要以单细胞悬浮液 (球形颗粒最佳) 为研究对象, 因此起初主要应用于哺乳动物细胞的常 规检测, 直到 $1970 \mathrm{~s}$ 末、1980s 初, 流式细胞仪才开始应用于水体浮游颗粒研究 ${ }^{[2-3]}$, 并迅速成为微型浮游生 物尤其是超微型浮游生物快速、准确的分析工具, 极大地促进了水生生态学的发展, 尤其在海洋水体应用广 泛 $^{[4-7]}$. 而且, 正是由于流式细胞仪的应用, 才导致了一些特定种群的发现, 如原绿球藻和最小的超微真核藻 Ostreococcus 等 ${ }^{[8.9]}$. 事实上, 在流式细胞计数应用于海洋浮游生物研究之前的很长一段时间内都是将原绿球 藻误认为是细菌而过高估计了细菌生物量. 目前国外已有一些关于流式细胞仪的文献综述, 对其原理、研究 进展等进行了详细介绍 ${ }^{[3,6,10-12]}$, 但是还没有专门针对微型浮游生物, 尤其是刚起步的原生动物及浮游病毒 的流式细胞仪应用综述. 本文针对不同类群的微型浮游生物, 详细介绍了流式细胞计数的具体应用原理、方 法及其进展, 旨在为水生生态学研究者们起到指导性作用.

\section{1 浮游藻类计数}

表 1 主要光合色素的光学特征

Tab. 1 Optical characteristics of the major photosynthetic pigments

\begin{tabular}{ccc}
\hline 色素类型 & 激发峰 $\lambda_{\mathrm{EX}} / \mathrm{nm}$ & 发射峰 $\lambda_{\mathrm{EM}} / \mathrm{nm}$ \\
\hline 叶绿素 & 465 & 685 \\
藻红素 & $540 \sim 570$ & $560 \sim 575$ \\
藻蓝素 & $620 \sim 640$ & 650 \\
苂光素 & 420 & 470 \\
\hline
\end{tabular}

浮游藻类细胞由于含有各种类型能够自发荧光 的光合色素, 如叶绿素 $\mathrm{a}$, 因而特别适用于流式细胞 检测, 而且根据叶绿素 $\mathrm{a}$ 红色苂光的存在与否, 可直 接将浮游藻类与其他无机颗粒或异养细胞区分开 来. 不同种类浮游藻类功能色素组成的不同主要表 现在激发光谱和发射光谱的差异上 (表 1$)^{[10]}$. 由于 光源限制,一般利用发射光谱区分不同类型藻类. 目 前流式细胞仪中多采用 $488 \mathrm{~nm}$ 蓝光激光源, 根据研 究需要还可额外定制添加光源 (如紫外激发光源).

一般在浮游藻类发射光谱中主要为叶绿素 $\mathrm{a} 、$ 藻红素和藻蓝素 3 种类型的菼光, 菼光素较少, 而类胡萝卜素 和其他微量色素则可能由于光合作用的能量转换而没有被观察到 ${ }^{[10]}$. 因此, 在水体中叶绿素 $\mathrm{a} 、$ 澡红素和藻 蓝素是主要苂光来源.

事实上, 从 $1980 \mathrm{~s}$ 流式细胞术应用于浮游澡类检测以来, 主要都是根据细胞大小及色素差异对一些特定 类群藻类进行识别 ${ }^{[13-14]}$. Trask 等对 8 种分属于 5 纲的不同纯培养藻类用流式细胞仪进行研究, 发现不同类 
型藻类由于大小和色素含量的不同, 其 FSC、SSC 及叶绿素 a 自发红色苂光的强度是不同的, 但由于存在着 大量的峰重叠区域, 仅用一种参数难以准确区分各种类群的藻; 尽管如此, 当将各参数结合起来时 (如 FSC vs SSC 或 SSC vs 叶绿素 a 等) 则能很好地区分不同种类的藻, 因为他们在二维图上的位置不同, 而参数的选 择则取决于藻的种类 ${ }^{[2]}$. 但是野外样品要比室内纯培养藻样复杂的多, 对于室内纯培养样品, 根据其在流式 二维或三维图上的位置就可确定藻种类型, 而野外样品藻的差异要大得多, 分布在流式图同一区域的细胞 可能是多种具有相同性质藻类的混合体, 尤其是大多数都为含有叶绿素 $\mathrm{a}$ 的真核藻类细胞 ${ }^{[14]}$. 但若某一类 群藻类含有特异性的光合色素或特殊结构时, 就可据此与其他类型藻类区分开来, 例如聚球藻 (Synechococcus)、原绿球藻 (Prochlorophytes)、具有钙质外壳的球石藻 (Coccolithophorids)、壳缝羽纹硅藻 (pennate diatoms) 和一些含藻红素的隐藻 (Cryptophytes) 等 ${ }^{[14-16]}$. 因此, 在研究野外样品时, 根据散射光和苂光信号, 流式 细胞仪一般只能将细胞区分为几个主要的光学性质相似的功能群, 如细胞粒径较大、叶绿素 a 含量丰富的 真核藻细胞和细胞粒径较小、藻蓝素或藻红素含量丰富的原核蓝藻细胞 ${ }^{[17-20]}$.

尽管如此, 由于水体中较大粒径的浮游藻类浓度普遍较低, 在相同的时间内通过检测区域的细胞数量 少,有的甚至低于流式细胞仪的检测限, 并且光谱特性同一性强, 基本都因含叶绿素 a 而发射红色苂 光 ${ }^{[14,21]}$, 而这些藻类在普通光学显微镜下也容易辨认, 因而其流式细胞应用受到限制. 相反, 相对于大型藻 类, 超微型浮游植物由于细胞浓度高, 足以达到流式细胞仪的检测范围, 而且由于细胞体积小, 形态特征不 明显, 普通显微观察难以辨认, 故更适合于流式细胞分析, 而目前流式细胞仪应用于浮游藻类研究也主要是 针对超微藻 ${ }^{[5,17-18]}$.

虽然流式细胞仪功能强大, 优势明显, 但是由于价格昂贵, 且属精密仪器, 一般的研究调查很难满足随 船携带的条件,无法对新鲜样品进行现场测定, 需要将样品固定后用液氮速冻保存, 带回实验室分析. 一般 常用的固定剂如鲁哥试剂、福尔马林(含甲醇)、乙醇等会对藻类色素荧光产生显著影响,因此不适用于流式 分析 ${ }^{[22]}$. 目前一般使用醛类固定剂如戊二醛、甲醛和多聚甲醛等 ${ }^{[23-25]}$ 用于流式细胞分析的样品保存,但是由 于市售的甲醛一般都含有甲醇, 而多聚甲醛不稳定, 需现配现用最好, 所以选用戊二醛为固定剂最方便实 用 ${ }^{[26]}$. Marie 等通过添加表面活性剂 Pluronic F68 优化了常规的固定方法, 可更好地减少固定引起的细胞 (尤其是真核细胞) 损失 ${ }^{[26]}$.

\section{2 浮游细菌计数}

$1980 \mathrm{~s}$ 流式细胞技术引人水生生态学领域初期, 由于浮游藻类所含光合色素是天然的荧光标记, 因此大 多研究都主要集中于浮游藻类的研究,如水体中两种主要的原核自养生物聚球藻和原绿球藻因含有不同的 光合色素, 利用流式细胞仪很容易区分. 但是, 分布在同一粒径范围内的异养细菌, 由于缺少易被识别的光 合色素, 流式细胞仪的应用受到阻碍, 发展也相对缓慢. 而实际上, 自然水体中异养细菌含量丰富, 浓度可达 $10^{6} \mathrm{cells} / \mathrm{ml}$ 的数量级, 非常适合流式细胞检测. 传统的细菌计数利用荧光染料如 DAPI 对其 DNA 进行染色, 然后用苂光显微镜观察计数, 这种染色法同样适用于流式细胞仪. 至 20 世纪初, 流式细胞仪已经发展成为 计数自养和异养细菌的标准方法. Robertson 等通过 DAPI 染色, 根据水体中细菌的种群丰度、大小和 DNA 含 量等特性, 首次利用流式细胞仪对自然水体中浮游细菌进行计数, 并给出了详细的染色方法和操作准则 ${ }^{[27]}$. 随后, Monger 等采用了另一种比 DAPI 背景荧光和 CV 值低且计数准确性更高的紫外激发荧光染料 Hoechst 33342 用于计数细菌 ${ }^{[28]}$. 尽管通过 DAPI 或 Hoechst 33342 染色后利用流式细胞仪计数的结果与菼光显微计 数的结果具有很好的一致性, 但是由于一些超微型光合自养原核细胞 (如原绿球藻) 与异养细菌的粒径大小 和 DAPI/Hoechst 33342-DNA 蓝色荧光都具有很大的重叠区, 而且用于区分异养细菌的原绿球藻叶绿素红色 苂光在紫外光激发下非常微弱,并且持续时间短, 无论是苂光显微镜还是流式细胞仪都难以辨别, 所以很难 将二者区分开来. 只有通过添加第二个 $488 \mathrm{~nm}$ 激发光源, 更好地激发叶绿素红色苂光, 才能将自异养细胞区 分开来, 从而准确地计数异养细菌 ${ }^{[28]}$.

苂光染料的选择一般取决于仪器可用的激发光源及样品类型. 采用紫外激发的苂光染料需要流式细胞 仪配置紫外激发光源, 因而应用受到限制. 而目前市售的流式细胞仪普遍采用 $488 \mathrm{~nm}$ 蓝光激发光源, 因此促 进了大量蓝光激发核酸染料的应用 (表 2), 这样只用一根激光就可区分自养和异养细菌. 由于 PI 和 EB 染料 
发射的红色荧光会对叶绿素苂光产生干扰, 因而不适用于自然水体原核生物的流式分析. 采用 YOYO-1、YOPRO-1 和 PicoGreen 等核酸染料虽然能取得较好的结果, 但是由于他们对离子的高敏感性, 而且需要辅助因 子如钾、柠檬酸盐和 EDTA 等的作用, 不适用于自然水体样品 ${ }^{[29]}$. 还有一些染料如 TOTO-1 和 TO-PRO-1 等 由于不渗透膜, 必须使用表面活性剂 (如 Triton X-100) 使其穿透细胞, 但是 Triton X-100 的使用会同时导致 叶绿素的损失, 从而无法区分异养细菌和原绿球藻 ${ }^{[30]}$. Lebaron 等对包括 SYTO-9、SYTO-11、SYTO-13、 SYTO-16、SYTO-BC、SYBR Green I 和 SYBR Green II 在内的 7 种蓝光激发的核酸染料进行比较研究, 这些染 料都可以用于完整的活细胞, 无需固定, 相反, 固定引起的细胞收缩会导致散射光测量偏差, 并且会降低 $\mathrm{SYTO}$ 类型染料的激发苂光 ${ }^{[31]}$; 此外, 对于不同类型的水体, 不同染料的苂光强度差异巨大: 对于淡水水样, SYTO-9、SYTO-16、SYBR Green I 和 SYBR Green II 的苂光强度更高, 而 SYTO-13、SYTO-11 的苂光强度较低, SYTO-9 的荧光强度最高, 但其属于试剂盒产品, 一般不单独销售; 相反, 对于海洋水样, SYBR Green I 和 SYBR Green II 具有更高的苂光强度 ${ }^{[31]}$. 目前, SYTO-13 和 SYBR Green I 是最适用于自然水体浮游细菌计数 的苂光染料, 其使用率也最高 ${ }^{[32-36]}$, 尤其是 SYTO-13. 值得注意的是, 不同的染料具体的染色浓度和染色时 间等也不同,已有很多研究者们对此作了详细验证 (表 2), 可为后来研究者提供参考.

表 2 已用于流式分析水体浮游细菌的核酸苂光染料

Tab. 2 Nucleic acid fluorochromes used to detect aquatic bacterioplankton by flow cytometry

\begin{tabular}{|c|c|c|c|c|c|c|}
\hline 染料类型 & 作用对象 & $\begin{array}{l}\text { 激发峰 } \\
\lambda_{\mathrm{EX}} / \mathrm{nm}\end{array}$ & $\begin{array}{c}\text { 发射峰 } \\
\lambda_{\mathrm{EM}} / \mathrm{nm}\end{array}$ & $\begin{array}{l}\text { 苂光 } \\
\text { 颜色 }\end{array}$ & 应用性能 & 参考文献 \\
\hline Chromomycin A3 & DNA & 340 & 470 & 蓝色 & 紫外激发, GC 特异性 & {$[37]$} \\
\hline DAPI & DNA & 358 & 461 & 蓝色 & 紫外激发, 半渗透膜, AT 特异性 & {$[27]$} \\
\hline Hoechst 33342 & DNA & 350 & 461 & 蓝色 & 紫外激发, 渗透膜, AT 特异性 & {$[28]$} \\
\hline Ethidium bromide( EB ) & DNA/RNA & 518 & 605 & 红色 & 不渗透膜 & {$[38]$} \\
\hline Propidium iodide( PI) & DNA/RNA & 536 & 617 & 红色 & 不渗透膜 & {$[39]$} \\
\hline TO-PRO-1 & DNA/RNA & 515 & 531 & 绿色 & 不渗透膜 & {$[30]$} \\
\hline TOTO-1 & $\mathrm{DNA} / \mathrm{RNA}$ & 514 & 533 & 绿色 & 不渗透膜, 海水中只染色 DNA & {$[30,33]$} \\
\hline YOYO-1 & DNA/RNA & 491 & 509 & 绿色 & 不渗透膜, 对离子敏感, 海水中只染色 DNA & {$[29,33]$} \\
\hline YO-PRO-1 & DNA/RNA & 491 & 509 & 绿色 & 不渗透膜, 对离子敏感 & [29] \\
\hline SYTO-9 & $\mathrm{DNA} / \mathrm{RNA}$ & 480 & 500 & 绿色 & 渗透膜 & {$[31]$} \\
\hline SYTO-11 & DNA/RNA & $508 \sim 510$ & $527 \sim 530$ & 绿色 & 渗透膜 & {$[31]$} \\
\hline SYTO-13 & DNA/RNA & 488 & 509 & 绿色 & 渗透膜,海水中只染色 DNA & {$[31-32]$} \\
\hline SYTO-16 & $\mathrm{DNA} / \mathrm{RNA}$ & 488 & 518 & 绿色 & 渗透膜 & {$[31]$} \\
\hline SYTO-17 & $\mathrm{DNA} / \mathrm{RNA}$ & 633 & 675 & 红色 & 渗透膜 & {$[40]$} \\
\hline SYTOX Green & 溶解 DNA & 504 & 523 & 绿色 & 不渗透膜 & {$[41]$} \\
\hline PicoGreen & 溶解 DNA & 480 & 520 & 绿色 & 对离子敏感 & {$[29,41]$} \\
\hline SYBR Green I & DNA( RNA) & 494 & 521 & 绿色 & 渗透膜, 与 DNA 具有更强的亲和性 & {$[31,34]$} \\
\hline SYBR Green II & RNA( DNA) & 494 & 521 & 绿色 & 渗透膜, 与 RNA 具有更强的亲和性 & {$[31]$} \\
\hline
\end{tabular}

利用流式细胞仪不仅可以快速、准确地对细菌总数进行计数, 而且可根据细胞相对大小和核酸含量将 细菌分成几个主要的亚类群, 分别进行计数. 对自然水体细菌的流式分析发现, 在流式散射光 vs 绿色荧光二 维图上可以明显区分出两种不同大小和核酸含量的细菌, 即低 DNA 含量 (LNA) 和高 DNA 含量 (HNA) 的细 菌 ${ }^{[30,35-36,42]}$. Garcia 等甚至提出了一种基于模型的聚类技术, 无需人工设门, 就可以自动将保存在流式文件 中的 LNA 和 HNA 细菌区分开来, 大大简化了后期的数据处理过程 ${ }^{[43]}$. 细菌核酸含量的差异可由多种原因 引起, 如 DNA 复制、细胞分裂、营养物质浓度等; 不同种类细菌也可能具有不同的 DNA 含量 ${ }^{[30]}$. 因而这些不 同核酸含量的细菌亚类群可能具有重要的生态意义. Li 等认为高 DNA 含量的细菌具有更高的生长势, 在浮 游植物丰富的水体占优势, 反之低 DNA 含量的细菌在浮游植物贫乏的水体占优势, 因此细菌受浮游植物影 响重大 ${ }^{[30]}$. Lebaron 等则发现细菌总生产力主要都是来自 HNA 细菌, 相对于细菌丰度, 细菌核酸含量更能表 征细菌的生长 ${ }^{[36]}$. 此外, 结合其他分子特异性苂光探针, 可更具体地针对不同种类的细菌进行计数, 而且还 
可以利用染料的膜通透性对细胞进行活性检测 ${ }^{[36,44-45]}$.

\section{3 原生动物计数}

相对于藻类和细菌, 原生动物的流式应用要复杂的多. 首先, 由于原生动物中没有可被流式细胞仪识别 的特征光合色素,如叶绿素、藻蓝素等,因而很难将其与其他颗粒区分开来;其次,水体中细菌等引起的背景 浓度过高, 难以有效区分原生动物细胞信号; 第三, 相对于细菌、病毒等超微型细胞颗粒, 原生动物粒径较 大, 在水体中的丰度相对较低, 几乎达到流式细胞仪的检测下限, 而流式细胞仪所测样品体积相对较小, 原 生动物一般很难被检测到. 所以流式细胞仪应用于原生动物计数一直受到限制. 直到近 10 年来, 才开始有 一些研究报道原生动物的流式检测, 而国内目前还没有相关报道. Zubkov 等在研究鞭毛虫对不同细菌的捕 食效率时, 通过 SYBR Green I 染色, 利用流式细胞仪测定了鞭毛虫的数量变化 ${ }^{[46]}$. 随后, Lindström 等建立 了一套利用流式细胞仪分析粒径更大的纯培养的纤毛虫等原生动物捕食速率的方法, 并与显微计数结果进 行了比较,获得了较好的一致性. 但是, 流式技术应用的前提是捕食者与被捕食者的细胞粒径相差很大,而 且杂质含量低,否则也很难将他们区分开来; 同时他们也比较了 3 种可被 $488 \mathrm{~nm}$ 蓝光激发的苂光染料 TO-PRO-1、YOYO-1 和 PicoGreen 的流式应用效果,并发现 TO-PRO-1 应用效果最好 ${ }^{[47]}$.

由于野外样品极其复杂, 所含杂质多, 因此目前有关流式细胞仪应用于原生动物的检测多为纯培养样 品, 而很少应用于自然水体. 此外, 原生动物最终产生的苂光信号要远远大于细菌产生的信号 (35 倍以上), 从而使原生动物信号位于象限外 ${ }^{[48]}$, 因此也不能将流式计数异养细菌的方法直接应用于原生动物计数. Rifà 等通过提高苂光信号的阈值并降低检测电压的方法, 使低苂光信号的颗粒如细菌等低于检测限, 而只 有较高荧光信号的颗粒 (多为异养鞭毛虫) 被检测到, 但此应用的主要限制条件就是水体中细菌与鞭毛虫的 比例不能高于 1000 , 否则, 高核酸含量细菌所产生的背景值过高, 仍会导致难以区分出异养原生动物 ${ }^{[48]}$. 而 实际野外水体中细菌与鞭毛虫的比例常常超过 1000 , 因此应用受到限制. Rose 等采用一种新的苂光染料 LysoTracker Green, 它对酸性细胞器如真核细胞中的营养液泡、溶酶体及叶绿体等具有亲和性, 而原核细胞 不具有这些细胞器, 从而将真核和原核细胞区分开来. 再通过自养细胞光合色素的自发苂光信号, 就可将自 养和异养真核细胞区分开来,从而鉴别出异养原生动物 ${ }^{[49]}$. 但是这种染料只能用于活细胞, 因为固定会破坏 细胞膜的势能, 导致苂光丢失. 当样品量较少时可采用这种方法, 但是当样品量很多时, 该方法的应用会受 到限制, 因为若不固定, 鞭毛虫等原生动物的丰度在几个小时内就会发生变化, 从而给测定结果造成影 响 ${ }^{[50]}$. 因此,应根据样品类型和研究目的选取合适的染料和检测方法.

另一方面, 自然水体中原生动物丰度很低, 如异养鞭毛虫的丰度通常只有 $10^{2} \sim 10^{3} \mathrm{cell} / \mathrm{s} / \mathrm{ml}$, 几乎达到 流式细胞仪的最低检测限, 虽然可通过富集浓缩的方法解决此问题, 但是由于原生动物细胞极其脆弱, 此方 法很可能造成巨大的细胞损失. Zubkov 等通过利用一种注射泵 (syringe pumps) 上样,大大提高了流式细胞 仪的分析流速, 可超过 $1.0 \mathrm{ml} / \mathrm{min}$, 这样在相同的时间内, 可分析更大体积的样品, 从而使更多的原生动物 细胞能够被检测到, 完美地解决了原生动物丰度过低的问题 ${ }^{[51-52]}$. 但是可能由于需要安装使用额外的洜, 此 方法并没有得到广泛应用 ${ }^{[50]}$. Christaki 等利用 SYBR Green I 染色, 结合前人的分析方法, 通过延长检测时 间、增加苂光信号的阈值并降低检测电压，同时对不同实验条件(固定剂种类、染色剂浓度、染色时间及流式 细胞仪设置等) 进行综合比较、验证和优化, 发现无需注射泵, 直接将流速提高到 $120 \sim 220 \mu \mathrm{l} / \mathrm{min}$, 同时增 加检测时间 (8 10 min), 就可以检测到 $10^{-2}$ cell $\mathrm{s} / \mathrm{ml}$ 数量级丰度的鞭毛虫. 此外, 研究还指出, 戊二醛固定 效果最好, 然后液氮速冻, $-80^{\circ} \mathrm{C}$ 保存最佳; 解冻后, 利用 SYBR Green I 染色, 终浓度为 $1: 10000$, 最佳染色时 间不短于 $10 \mathrm{~min}$, 而且无需添加柠檬酸钾等就能达到很好的染色效果. 他们提出的这一套异养鞭毛虫流式 计数方法更为方便简单, 相信会得到广泛应用 ${ }^{[50]}$.

\section{4 浮游病毒计数}

由于病毒颗粒太小, Porter 等指出几乎不可能利用传统的流式细胞仪进行检测 ${ }^{[53]}$. 但是, 随着一些高性 能绿色苂光核酸染料如 SYBR Green I 、SYTO 13 和 TO-PRO-1 等的应用,流式细胞仪应用于浮游病毒的检 测也成为可能. 这些苂光染料在 $488 \mathrm{~nm}$ 激光激发下具有更高的量子产率, 可发射更强的绿色苂光, 大大提高 
了流式细胞仪的检测限. Marie 等通过 SYBR Green I 染色首次利用标准流式细胞仪成功地检测和计数了海 洋中的浮游病毒, 并且与透射电镜和荧光显微镜的计数结果具有很高的相关性 ${ }^{[54]}$. Brussaard 等发现大多数 不同形态特征和基因大小的病毒都可利用流式细胞仪进行检测 ${ }^{[55]}$. 结合 SYBR Green I 染色, 流式细胞仪不 仅可用于室内纯培养病毒样品检测 ${ }^{[56-57]}$, 而且还被成功地应用于自然环境样品检测 ${ }^{[58-62]}$.

影响流式细胞仪病毒检测的因素有很多, 例如固定、保存的方法, 稀释液的类型, 染料的种类和浓度以 及孵育温度等 ${ }^{[54]}$. 研究发现, 戊二醛固定效果比多聚甲醛更加稳定 ${ }^{[55]}$, 在 $0.1 \% \sim 1.0 \%$ 范围内不同浓度戊 二醛固定对病毒计数结果并无显著影响 ${ }^{[54]}$. 但是无论是多聚甲醛还是戊二醛, 固定后若仅保存于 $4^{\circ} \mathrm{C}$, 随时 间推移, 病毒浓度均逐渐减少, 这可能是由于聚集物形成或核酸酶对病毒的降解所致, 所以应液氮速冻后, $-80^{\circ} \mathrm{C}$ 低温保存 ${ }^{[54]}$. 其次, 由于浮游病毒是水体中数量最大的生命颗粒, 可达 $10^{10} \mathrm{~L}^{-1}$, 是细菌的 $5 \sim 25$ 倍, 为防止流式分析时巧合事件 (由于样品浓度过高, 常常两个或多个颗粒同时通过检测区域, 而仪器只能将多 个颗粒记为一个事件, 从而低估样品浓度, 称为巧合事件) 的发生, 必须将样品稀释后再进行分析. Marie 等 利用培养基、 $0.2 \mu \mathrm{m}$ 过滤的海水和 TE 缓冲液 3 种溶液分别对样品进行稀释, 发现利用 TE 缓冲液稀释效果 最好, 培养基和 $0.2 \mu \mathrm{m}$ 过滤的海水稀释要比用 TE 缓冲液稀释后的检测结果低 $5 \sim 6$ 倍, 而且用培养基稀释 所产生的背景值更高 ${ }^{[54]}$. 染料的种类和浓度对计数结果也有较大影响. Chen 等利用流式细胞仪对聚球藻病 毒进行检测, 发现 SYBR Gold 比 SYBR Green I 染色效果更好 ${ }^{[59]}$. 而 Brussaard 等对 SYBR Green I 、SYBR Green II、OliGreen 和 PicoGreen 4 种染料进行比较, 发现 SYBR Green I 效果最好, 产生的绿色苂光最强 ${ }^{[55]}$, 它也是目前使用最多的染料. 此外, 由于病毒核酸被衣壳包被, 不能立即与 SYBR-I 或其他核酸染料结合, 从 而使检测结果被低估, 通过加热或添加表面活性剂如 Triton X-100 可使病毒衣壳变性, 使染料穿透 ${ }^{[54]}$. 所以 通常分析前, 将样品加热到 $80^{\circ} \mathrm{C}$ 可显著增强染色效果 ${ }^{[63]}$. 由于影响因素众多, 不同研究者们所采用的方法 常常不同. Brussaard 等通过对不同实验条件进行比较, 总结了流式细胞仪计数病毒的最佳条件, 即: $0.5 \%$ 终 浓度的戊二醛于 $4^{\circ} \mathrm{C}$ 固定 $5 \sim 30 \mathrm{~min}$, 液氮速冻后 $-80^{\circ} \mathrm{C}$ 低温保存; 利用 $\mathrm{TE}$ 缓冲液 $(\mathrm{pH}=8)$ 稀释, $\mathrm{SYBR}$ Green I 染色, 染料终浓度为 $5 \times 10^{-5}$, 然后暗处 $80^{\circ} \mathrm{C}$ 睬育 $10 \mathrm{~min}$, 最后冷却 $5 \mathrm{~min}$, 就可上样进行流式 分析 ${ }^{[63]}$.

病毒粒径只有几十到几百纳米, 多数都小于激发光波长, 因此 SSC 并不能表征颗粒的大小和粒度, 而且 研究还发现染色后绿色苂光强度与病毒基因大小也并无显著线性相关关系 ${ }^{[55]}$. 尽管如此, SSC 和绿色苂光 信号强度对于不同病毒类型的区分仍非常有用. 根据绿色荧光信号的强度, 流式细胞仪可至少区分出自然 水体中两个不同类群的浮游病毒: V - I 型和 V - I 型. V - I 型病毒绿色荧光最强, 粒径更大; 而 V - II 型病毒 能全部通过 $0.2 \mu \mathrm{m}$ 孔径滤膜, 粒径相对较小; 在数量上, V-II 型病毒是 V-I 型病毒的 $4 \sim 10$ 倍, 与自养和 异养细胞的比例相当, 表明 V - I 型病毒很可能是浮游植物 (尤其是真核细胞) 病毒, 而 V - II 型病毒则可能是 丰度更高的异养细菌病毒 ${ }^{[54]}$.

\section{5 应用展望}

目前流式细胞仪在微型浮游生物方面的应用仍以计数为主. 但其在微生物生态学领域的应用远不止于 此, 可以说其应用具有相当的灵活性, 只要颗粒能够发射苂光或通过某种方法使其发射苂光就能被流式细 胞仪检测. 除了对这些微型浮游生物进行常规的检测计数外, 流式细胞仪还可以测定微生物中 DNA 和 RNA 的含量, 并且能进行细胞周期分析 ${ }^{[64]}$; 结合分子特异性苂光探针, 进行原位杂交, 利用流式细胞仪可对具体 到属种水平的不同微生物类群进行分析 ${ }^{[44,65-66]}$.

此外, 流式细胞仪最强大也是其特有的功能在于分选, 它能够将其检测出来的细胞亚类群分选出来, 供 进一步深人研究, 如利用流式细胞仪分选出来的细胞进行纯培养 ${ }^{[67]}$, 还可以结合分子生物学技术, 进行系统 进化分析 ${ }^{[19]}$. 甚至可以将单个细胞分选出来进行分析 ${ }^{[68]}$, 这必然也会成为一种新的研究趋势.

值得注意的是, 利用流式细胞仪区分出来的细胞类群大都并不是系统进化阶元上的某一具体的分类类 群, 它们只是通过细胞光学性质区分出的具有相似性质 (如大小、色素含量等) 的某一生态功能群, 如富含叶 绿素的超微真核藻、藻蓝素细胞、藻红素细胞以及 LNA 和 HNA 等, 这既是流式细胞仪应用的一大限制, 也是 一种优势. 因为这些细胞类群也具有重要的生态意义, 甚至有学者提出了 “流式细胞多样性” 的概念, 用以表 
征某自然水体中流式细胞仪分辨出细胞类群的多样性 ${ }^{[30]}$. 已有研究表明, 叶绿素 $\mathrm{a}$ 和初级生产力较高的水 体中流式细胞多样性和均匀度更高 ${ }^{[69]}$. Schiaffino 等发现水体营养状态对超微型浮游生物的流式细胞多样 性影响重大, 藻红素细胞在塞营养和透明度较高的水体中更占优势, 而随着叶绿素浓度的升高, 藻蓝素细胞 和超微真核藻取代藻红素细胞成为竞争优势种; 更重要的是, 利用流式细胞仪与变性梯度凝胶电泳 (DGGE) 获得了相同的多样性结果 ${ }^{[70]}$. 相信对 “流式细胞多样性” 的记录及其分析研究可进一步促进流式细胞仪的 应用. 另一方面, 也可通过流式细胞仪的分选功能, 将其区分出来的细胞类型分选出来, 再结合苂光显微镜 进行种属鉴定或通过分子生物学技术进行系统发育分析, 同时建立数据库,这样在日后的研究中就可利用 已建立的数据库对野外样品进行具体的分类计数. 此外,近年来新开发的流式影像术 (FlowCAM) 通过结合 流式细胞术和显微镜技术, 不仅实现了浮游生物的自动分类, 更显著提高了样品的分析效率 ${ }^{[71]}$; 结合光片照 明显微技术, 甚至可以获得 $3 \mathrm{D}$ 图像, 大大提高了分辨率和精确度 ${ }^{[72]}$.

传统的流式细胞仪更加适用于分析较小的细胞 ( $<15 \mu \mathrm{m})$, 而对于体积较大的浮游生物细胞例如原生 动物等的应用常常受到限制, 而且由于湖泊、河流等淡水样品所含杂质较多、背景复杂, 为防止堵塞流式细 胞仪喷嘴, 通常还需要经过预过滤处理, 非常不利于传统流式细胞仪的应用. 但是, 随着技术的发展, 一些功 能更加强大的新型流式细胞仪不断被开发出来, 为浮游生物的监测提供了新的手段. 例如便携式浮游植物 分析流式细胞仪 (CytoSense)、在线监测型流式细胞仪 (Cytobouy) 和可在水下使用的自动流式细胞仪 (FlowCytobot) 等, 他们实现了对微型浮游生物进行连续定量、实时原位的监测 ${ }^{[73-75]}$. 相信技术的革新及与其他技 术的联用将会使流式细胞术越来越广泛地应用于水体浮游生物检测方面的研究.

\section{6 参考文献}

[ 1 ] Azam F, Fenchel T, Field JG et al. The ecological role of water-column microbes in the sea. Marine Ecology-Progress Series, $1983, \mathbf{1 0}(3)$ : 257-263.

[ 2 ] Trask B, van den Engh G, Elgershuizen J. Analysis of phytoplankton by flow cytometry. Cytometry, 1982, 2 (4): 258-264.

[ 3 ] Legendre L, Yentsch CM. Overview of flow cytometry and image analysis in biological oceanography and limnology. Cytometry, $1989, \mathbf{1 0}(5): 501-510$.

[ 4 ] Olson R, Vaulot D, Chisholm S. Marine phytoplankton distributions measured using shipboard flow cytometry. Deep Sea Research Part A : Oceanographic Research Papers, 1985, 32(10) : 1273-1280.

[ 5 ] Li WKW. Primary production of prochlorophytes, cyanobacteria, and eukaryotic ultraphytoplankton: measurements from flow cytometric sorting. Limnology and Oceanography, 1994, 39(1) : 169-175.

[ 6 ] Legendre L, Courties C, Troussellier M. Flow cytometry in oceanography 1989-1999: environmental challenges and research trends. Cytometry, 2001, 44(3): 164-172.

[ 7 ] 郭沛涌, 朱荫泦. 流式细胞术在富营养淡水湖泊微型浮游植物细胞中的应用. 细胞生物学杂志, 2004,26(2): 204-206.

[ 8 ] Chisholm SW, Olson RJ, Zettler ER et al. A novel free-living prochlorophyte abundant in the oceanic euphotic zone. Nature, $1988,334: 340-343$.

[ 9 ] Courties C, Vaquer A, Trousselier M et al. Smallest eukaryotic organism. Nature, 1994, 370(6487) : 255.

[10] Phinney D, Cucci T. Flow cytometry and phytoplankton. Cytometry, 1989, 10(5): 511-521.

[11] Marie D, Simon N, Vaulot D. Phytoplankton cell counting by flow cytometry. In: Andersen RA ed. Algal culturing techniques. New York: Academic Press, 2005 : 253-268.

[12] Sosik HM, Olson RJ, Armbrust EV. Flow cytometry in phytoplankton research. In: Suggett DJ, Prasil O, Borowitzka MA eds. Chlorophyll a fluorescence in aquatic sciences: methods and applications. New York: Springer, 2011 : 171-185.

[13] Veldhuis MJ, Kraay GW. Application of flow cytometry in marine phytoplankton research: current applications and future perspectives. Scientia Marina, 2000, 64(2): 121-134.

[14] Olson R, Zettler E, Anderson O. Discrimination of eukaryotic phytoplankton cell types from light scatter and autofluorescence properties measured by flow cytometry. Cytometry, 1989, 10(5) : 636-643.

[15] Burkill P, Leakey R, Owens N et al. Synechococcus and its importance to the microbial foodweb of the northwestern Indian 
Ocean. Deep Sea Research Part II: Topical Studies in Oceanography, 1993 , 40(3) : 773-782.

[16] Olson RJ, Zettler ER, DuRand MD. Phytoplankton analysis using flow cytometry. In: Kemp PF, Sheer BF, Sheer EB et al ed. Handbook of methods in aquatic microbial ecology. Boca Raton: Lewis Publishers, 1993 : 175-186.

[17] Crosbie ND, Teubner K, Weisse T. Flow cytometric mapping provides novel insights into the seasonal and vertical distributions of freshwater autotrophic picoplankton. Aquatic Microbial Ecology, 2003, 33(1) : 53-66.

[18 ] Winder M. Photosynthetic picoplankton dynamics in Lake Tahoe: temporal and spatial niche partitioning among prokaryotic and eukaryotic cells. Journal of Plankton Research, 2009, 31(11) : 1307-1320.

[19] Marie D, Shi XL, Rigaut-Jalabert F et al. Use of flow cytometric sorting to better assess the diversity of small photosynthetic eukaryotes in the English Channel. FEMS Microbiology Ecology, 2010, 72(2) : 165-178.

[20］李胜男,史小丽,谢薇薇等. 长江中下游湖泊超微型真核藻类遗传多样性研究. 环境科学, 2013,34(9) : 3416-3422.

[21] Hofstraat J, van Zeijl W, de Vreeze M et al. Phytoplankton monitoring by flow cytometry. Journal of Plankton Research, 1994, 16(9) : 1197-1224.

[22] Marie D, Brussaard C, Partensky F et al. Flow cytometric analysis of phytoplankton, bacteria and viruses. Current Protocols in Cytometry, 1999, $11: 1-15$.

[23 L Lazinsky D, Sickogoad L. Paraformaldehyde-glutaraldehyde as a routine phytoplankton fixative. Micron, 1979, 10 (1): 49-50.

[24] Vaulot D, Courties C, Partensky F. A simple method to preserve oceanic phytoplankton for flow cytometric analyses. Cytometry, $1989, \mathbf{1 0}(5)$ : 629-635.

[25] Landry M, Kirshtein J, Monger B. Quantitative enumeration of paraformaldehyde preserved Prochlorococcus by flow cytometry. Signal Noise, 1993, 6:3.

[26] Marie D, Rigaut-Jalabert F, Vaulot D. An improved protocol for flow cytometry analysis of phytoplankton cultures and natural samples. Cytometry Part A, 2014, 85(11) : 962-968.

[27] Robertson BR, Button DK. Characterizing aquatic bacteria according to population, cell size and apparent DNA content by flow cytometry. Cytometry, 1989, 10(1): 70-76.

[28] Monger BC, Landry MR. Flow cytometric analysis of marine bacteria with Hoechst 33342. Applied and Environmental Microbiology, 1993, 59(3) : 905-911.

[29] Marie D, Vaulot D, Partensky F. Application of the novel nucleic acid dyes YOYO-1, YO-PRO-1, and PicoGreen for flow cytometric analysis of marine prokaryotes. Applied and Environmental Microbiology, 1996, 62 (5) : 1649-1655.

[30 ] Li W, Jellett J, Dickie P. DNA distributions in planktonic bacterial stained with TOTO or TO-PRO. Limnology and Oceanography, 1995, 40(8): 1485-1495.

[31] Lebaron P, Parthuisot N, Catala P. Comparison of blue nucleic acid dyes for flow cytometric enumeration of bacteria in aquatic systems. Applied and Environmental Microbiology, 1998, 64(5) : 1725-1730.

[32] Giorgio PAD, Bird DF, Prairie YT et al. Flow cytometric determination of bacterial abundance in lake plankton with the green nucleic acid stain SYTO 13. Limnology and Oceanography, 1996, 41(4) : 783-789.

[33 ] Guindulain T, Comas J, Vives-Rego J. Use of nucleic acid dyes SYTO-13, TOTO-1, and YOYO-1 in the study of Escherichia coli and marine prokaryotic populations by flow cytometry. Applied and Environmental Microbiology, 1997, 63(11) : $4608-4611$.

[34] Marie D, Partensky F, Jacquet S et al. Enumeration and cell cycle analysis of natural populations of marine picoplankton by flow cytometry using the nucleic acid stain SYBR Green I. Applied and Environmental Microbiology, 1997, 63(1): 186-193.

[35] Troussellier M, Courties C, Lebaron P et al. Flow cytometric discrimination of bacterial populations in seawater based on SYTO 13 staining of nucleic acids. FEMS Microbiology Ecology, 1999, 29(4) : 319-330.

[36] Lebaron P, Servais P, Agogué H et al. Does the high nucleic acid content of individual bacterial cells allow us to discriminate between active cells and inactive cells in aquatic systems? Applied and Environmental Microbiology, 2001, 67(4) : $1775-1782$.

[37] Boye E, Steen HB, Skarstad K. Flow cytometry of bacteria: a promising tool in experimental and clinical microbiology. Journal of General Microbiology, 1983, 129(4) : 973-980.

[38 ] Pinder A, Purdy P, Poulter S et al. Validation of flow cytometry for rapid enumeration of bacterial concentrations in pure 
cultures. Journal of Applied Bacteriology, 1990, 69(1): 92-100.

[39] Miller JS, Quarles JM. Flow cytometric identification of microorganisms by dual staining with FITC and PI. Cytometry, $1990,11(6): 667-675$.

[40 ] Comas J, Vives-Rego J. Assessment of the effects of gramicidin, formaldehyde, and surfactants on Escherichia coli by flow cytometry using nucleic acid and membrane potential dyes. Cytometry, 1997, 29(1) : 58-64.

[41] Veldhuis MJ, Cucci TL, Sieracki ME. Cellular DNA content of marine phytoplankton using two new fluorochromes: taxonomic and ecological implications. Journal of Phycology, 1997, 33(3) : 527-541.

[42] Bouvier T, Del Giorgio PA, Gasol JM. A comparative study of the cytometric characteristics of High and Low nucleic-acid bacterioplankton cells from different aquatic ecosystems. Environmental Microbiology, 2007, 9(8) : 2050-2066.

[43] Garcia FC, Lopez-Urrutia A, Moran XAG. Automated clustering of heterotrophic bacterioplankton in flow cytometry data. Aquatic Microbial Ecology, 2014, 72(2) : 175-185.

[44] Zoetendal EG, Ben-Amor K, Harmsen HJ et al. Quantification of uncultured Ruminococcus obeum-like bacteria in human fecal samples by fluorescent in situ hybridization and flow cytometry using 16S rRNA-targeted probes. Applied and Environmental Microbiology, 2002, 68(9): 4225-4232.

[45] Sekar R, Fuchs BM, Amann R et al. Flow sorting of marine bacterioplankton after fluorescence in situ hybridization. Applied and Environmental Microbiology, 2004, 70(10): 6210-6219.

[46] Zubkov MV, Zöllner E, Jürgens K. Digestion of bacterial macromolecules by a mixotrophic flagellate, Ochromonas sp. , compared with that by two heterotrophic flagellates, Spumella pudica and Bodo saltans. European Journal of Protistology, $2001,37(2): 155-166$.

[47] Lindström ES, Weisse T, Stadler P. Enumeration of small ciliates in culture by flow cytometry and nucleic acid staining. Journal of Microbiological Methods, 2002, 49(2): 173-182.

[48] Rifà TG, Latatu A, Ayo B et al. Flow cytometric detection and quantification of heterotrophic nanoflagellates in enriched seawater and cultures. Systematic and Applied Microbiology, 2002, 25(1) : 100-108.

[49] Rose JM, Caron DA, Sieracki ME et al. Counting heterotrophic nanoplanktonic protists in cultures and aquatic communities by flow cytometry. Aquatic Microbial Ecology, 2004, 34(3) : 263-277.

[50] Christaki U, Courties C, Massana R et al. Optimized routine flow cytometric enumeration of heterotrophic flagellates using SYBR Green I. Limnology and Oceanography-Methods, 2011, 9: 329-339.

[51] Zubkov MV, Burkill PH. Syringe pumped high speed flow cytometry of oceanic phytoplankton. Cytometry Part A, 2006, 69(9) : 1010-1019.

[52] Zubkov MV, Burkill PH, Topping JN. Flow cytometric enumeration of DNA-stained oceanic planktonic protists. Journal of Plankton Research, 2007, 29(1) : 79-86.

[53] Porter J, Deere D, Hardman M et al. Go with the flow-use of flow cytometry in environmental microbiology. FEMS Microbiology Ecology, 1997, 24(2) : 93-101.

[54] Marie D, Brussaard CPD, Thyrhaug R et al. Enumeration of marine viruses in culture and natural samples by flow cytometry. Applied and Environmental Microbiology, 1999, 65(1): 45-52.

[55] Brussaard CPD, Marie D, Bratbak G. Flow cytometric detection of viruses. Journal of Virological Methods, 2000, 85(1): $175-182$.

[56] Brussaard CPD, Marie D, Thyrhaug R et al. Flow cytometric analysis of phytoplankton viability following viral infection. Aquatic Microbial Ecology, 2001, 26(2) : 157-166.

[57] Brussaard CPD, Thyrhaug R, Marie D et al. Flow cytometric analysis of viral infection in two marine phytoplankton species, Micromonas pusilla (Prasinophyceae) and Phaeocystis pouchetii( Prymnesiophyceae). Journal of Phycology, 1999, $35(5)$ : $941-948$.

[58 ] Castberg T, Larsen A, Sandaa RA et al. Microbial population dynamics and diversity during a bloom of the marine coccolithophorid Emiliania huxleyi ( Haptophyta). Marine Ecology Progress Series, 2001, 221 :39-46.

[59] Chen F, Lu J, Binder BJ et al. Application of digital image analysis and flow cytometry to enumerate marine viruses stained with SYBR Gold. Applied and Environmental Microbiology, 2001, 67(2) : 539-545.

[60] Larsen A, Castberg T, Sandaa RA et al. Population dynamics and diversity of phytoplankton, bacteria and viruses in a seawater enclosure. Marine Ecology Progress Series, 2001, 221 :47-57. 
[61] Li WKW, Dickie PM. Monitoring phytoplankton, bacterioplankton, and virioplankton in a coastal inlet (Bedford Basin) by flow cytometry. Cytometry, 2001, 44(3): 236-246.

[62] Wilson WH, Tarran G, Zubkov MV. Virus dynamics in a coccolithophore-dominated bloom in the North Sea. Deep Sea Research Part II : Topical Studies in Oceanography, 2002, 49(15) : 2951-2963.

[63] Brussaard CPD. Optimization of procedures for counting viruses by flow cytometry. Applied and Environmental Microbiolo$g y, 2004,70(3): 1506-1513$.

[64] Binder BJ, Chisholm SW. Relationship between DNA cycle and growth rate in Synechococcus sp. strain PCC 6301. Journal of Bacteriology, 1990, 172(5) : 2313-2319.

[65] Amann RI, Ludwig W, Schleifer KH. Phylogenetic identification and in situ detection of individual microbial cells without cultivation. Microbiological Reviews, 1995, 59(1) : 143-169.

[66] Biegala IC, Not F, Vaulot D et al. Quantitative assessment of picoeukaryotes in the natural environment by using taxonspecific oligonucleotide probes in association with tyramide signal amplification-fluorescence in situ hybridization and flow cytometry. Applied and Environmental Microbiology, 2003, 69(9) : 5519-5529.

[67 Crosbie ND, Pöckl M, Weisse T. Rapid establishment of clonal isolates of freshwater autotrophic picoplankton by singlecell and single-colony sorting. Journal of Microbiological Methods, 2003, 55(2) : 361-370.

[68 ] Collier JL. Flow cytometry and the single cell in phycology. Journal of Phycology, 2000, 36(4) : 628-644.

[69] Li WKW. Cytometric diversity in marine ultraphytoplankton. Limnology and Oceanography, 1997, 42 (5) : 874-880.

[70 ] Schiaffino MR, Gasol JM, Izaguirre I et al. Picoplankton abundance and cytometric group diversity along a trophic and latitudinal lake gradient. Aquatic Microbial Ecology, 2013, 68(3) : 231-250.

[71 ] Álvarez E, López-Urrutia Á, Nogueira E. Improvement of plankton biovolume estimates derived from image-based automatic sampling devices: application to FlowCAM. Journal of Plankton Research, 2012, 34(6) : 454-469.

[72] Wu J, Li J, Chan RKY. A light sheet based high throughput 3D-imaging flow cytometer for phytoplankton analysis. Optics Express, 2013, 21(12) : 14474-14480.

[73] Thyssen M, Grégori GJ, Grisoni JM et al. Onset of the spring bloom in the northwestern Mediterranean Sea: influence of environmental pulse events on the in situ hourly-scale dynamics of the phytoplankton community structure. Frontiers in Microbiology, 2014, $5: 387$.

[74] Pomati F, Jokela J, Simona M et al. An automated platform for phytoplankton ecology and aquatic ecosystem monitoring. Environmental Science and Technology, 2011, 45(22) : 9658-9665.

[75] Campbell L, Henrichs DW, Olson RJ et al. Continuous automated imaging-in-flow cytometry for detection and early warning of Karenia brevis blooms in the Gulf of Mexico. Environmental Science and Pollution Research, 2013, 20(10) : 68966902. 